\title{
Design, Analysis, and System-Level Simulation of a Micromachined Resonant Charge Sensor with Microleverage Mechanisms
}

\author{
Jiuxuan Zhao, Hong Ding, Wen Wang ${ }^{1}$ and Jin Xie* \\ The State Key Laboratory of Fluid Power Transmission and Control, Zhejiang University, \\ Hangzhou 310027, People's Republic of China \\ 'School of Mechanical Engineering, Hangzhou Dianzi University, \\ Hangzhou 310018, People's Republic of China
}

(Received March 30, 2015 accepted June 23, 2015)

Key words: resonant charge sensor, micro-electromechanical systems, microlevers, sensitivity enhancement

In this paper, a micromechanical electrostatic resonant charge sensor with responsivity enhanced by introducing dual single-stage microleverage mechanisms (microlevers) is presented. The sensor is based on the mechanism of detecting the resonant frequency shift of a single-crystal silicon double-ended tuning fork (DETF) element due to the axial compression generated by the additional charge and transformed by the microlevers. An analytic model comprising both mechanical and electrical subsystems was derived. The system-level simulation was carried out to optimize the critical dimensions and responsivity that coincides with the theoretical solution. The resonator has a nominal resonant frequency of $166 \mathrm{kHz}$ and the amplification factor is more than 8 . Simulation results suggest that the responsivity of the charge sensor is $1.38 \times 10^{-3} \mathrm{~Hz} / \mathrm{fC}^{2}$. Finally, the device was fabricated in a commercial standard siliconon-insulator (SOI) micromachining process to reduce the parasitic capacitance more effectively by back-side release.

\section{Introduction}

Charge sensors, also known as electrometers, have found a large range of applications, including the investigation of atmospheric particle dynamics, ${ }^{(1)}$ isotope ratio measurements, ${ }^{(2)}$ ultrafine particle detection, ${ }^{(3)}$ and even space exploration. ${ }^{(4)}$ Current conventional electrometers have good resolution but are bulky and difficult to integrate with other systems. At present, cryogenically cooled single-electron transistors with reported noise levels of $10^{-5} \mathrm{e} / \sqrt{\mathrm{Hz}}$, operating at a temperature of about $50 \mathrm{mK}$, are

"Corresponding author: e-mail: xiejin@zju.edu.cn 
considered state-of-the-art electrometers. ${ }^{(5)}$ Micro-electromechanical system (MEMS) technology was developed in the last decade to address the need for charge measurement. The modulation of input capacitance for the measurement of charge with a resolution of 28 electrons at a $0.3 \mathrm{~Hz}$ bandwidth was demonstrated using a microscale vibratingreed capacitor to move the detection bandwidth away from the $1 / f$ noise regime. ${ }^{(6)}$ The concept was extended, achieving a higher resolution of $6 e / \sqrt{\mathrm{Hz}}$ at room temperature and ambient pressure, by reducing parasitic capacitances. ${ }^{(7)}$ An electrometer based on the vibrating capacitance for the detection of aerosol particles which reported a minimum detectable $10 \mathrm{~nm}$ diameter particle density of $400 \mathrm{~cm}^{-3}$ within an airflow of $1.0 \mathrm{~L} / \mathrm{min}$ has been presented. ${ }^{(3)}$ Charge sensing can also be indirectly realized through the measurement of the electrostatic force generated across the plates of a capacitor. Nanoscale mechanical electrometers based on the resonance frequency shift were reported to have a subelectron resolution at a temperature of about $4.5 \mathrm{~K}^{\left({ }^{(8,9)}\right.}$ A MEMS double-ended tuning fork (DETF) electrometer modulated by axial strain has been demonstrated, ${ }^{(10)}$ yielding a minimum measurable charge of $4 \mathrm{fC}$. The sensitivity can be further enhanced by introducing mechanical amplifiers to magnify the electrostatic force.

Mechanical amplifiers can be classified into elaborate displacement-amplifying compliant mechanisms (DaCMs) ${ }^{(11)}$ and simple compliant microleverage mechanisms (microlevers) ${ }^{(12-14)}$ used to amplify displacement or force. Microlevers have already been used in previous studies for various microelectromechanical systems. ${ }^{(15,16)}$ However, no study has been performed to apply such mechanisms in charge sensors. In this study, we aim to increase the sensitivity of a resonant charge sensor by using microlevers.

The original systematical study on microlevers has been reported.(14) With fabrication technology constraints, the design of microlevers is different from that of conventional levers in the macroworld. Compliant mechanisms are mechanical devices without joints that achieve force or motion transformation by taking advantage of elastic deformation. Owing to elastic energy consumption, the amplification effect of microlevers is inferior to that of conventional levers.

In this paper, we report a resonant charge sensor based on a DETF element and introduce dual single-stage microlevers. The charge to be measured is added to the plates of double input capacitors, which induce electrostatic force that is then transformed by the microlevers. The output frequency of the sensor is modulated by the axial compressive stress amplified by the microlevers. An analytic model used to describe sensor sensitivity is derived and the relationship between output frequency shift and input charge is determined to be quadratic. The proposed charge sensor has advantages of simple implementation, quasi-digital nature of the signal, and sensitivity enhanced by using dual microlevers. The mechanical amplifier does not add significant Brownian noise and not affect the electronic noise level of the interface circuitry.

\section{Design and Theory}

The schematic drawing of a resonant charge sensor with microlevers is shown in Fig. 1. The sensor has a DETF resonator formed by two parallel beams or tines clamped to both tethers. The DETF element is anchored on one side and attached to double single- 


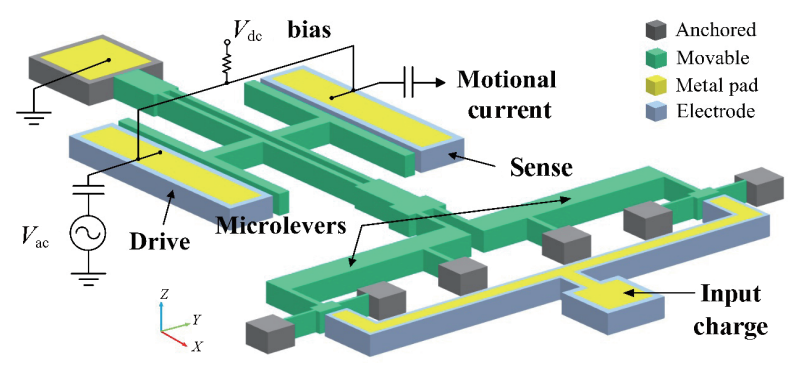

Fig. 1. (Color online) Schematic of the resonant charge sensor with dual microlevers.

stage microlevers on the other side. Driving and sensing electrodes are attached to both tines to drive the resonator to vibrate and sense the motional current, respectively. The DETF resonator includes two important low vibration modes, namely, in-phase and outof-phase modes. Generally, the in-phase mode has a lower mode frequency than the outof-phase mode owing to the longer effective beam length of the in-phase mode. The outof-phase vibration mode has the advantage of realizing a higher $\mathrm{Q}$ value by mutually canceling opposing stress waves in the coupling end of tines. Therefore, the DETF resonator working under the out-of-phase mode was used in this device instead of a simple clamped-clamped beam resonator.

A single-stage microlever is comprised of four major parts: input system, output system, pivot and lever arm, as schematically shown in Fig. 2, in which the lever arm is the rigid part, while the pivot and connections are the flexible elements. Because a backside trench is created to release the structure in fabrication, all the anchors are placed in boundary zones. As a result, the microlever was designed with the pivot lying between the input and the output, and the pivot and output on the same side as shown in Figs. 1 and 2. The input plate is supported by two narrow, long beams to avoid the lever arm in suspension, which increase the stability of the whole structure. Double charge input capacitors are formed by four plates at the input of the microlevers.

The schematic in Fig. 2 illustrates the derivation of the amplification factor of the microlever in the proposed design. The pivot and lever arm deformations due to an input force are taken into account, while the deformation of the input beam is neglected under the small deflection condition. The horizontal displacements cancel each other out since the double microlevers are distributed symmetrically. To investigate the amplification factor, the following four assumptions have been considered:(14) (i) the lever arm remains straight under loading, (ii) the rotation angle is only determined by bending moments, (iii) the vertical displacement is only caused by vertical force, and (iv) all discussions are within the regime of linear elasticity. The microlever shapes before and after loading are shown in Fig. 2(a), and the simplified model used to derive the amplification factor is shown in Fig. 2(b).

When an input force is applied, the overall deflections of the microlever and the directions of all the forces and moments are shown in Fig. 2. According to force and moment equilibrium, the equations of the microlever are given by 


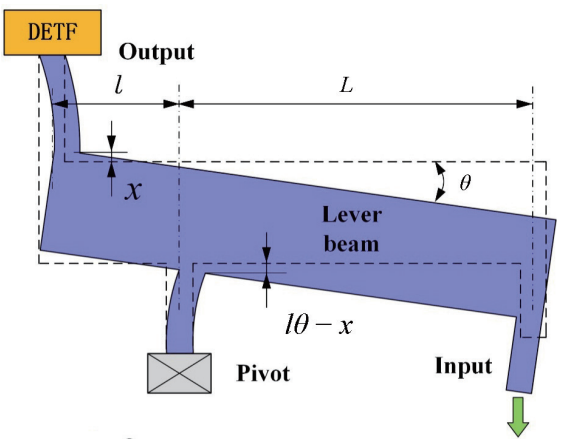

(a)

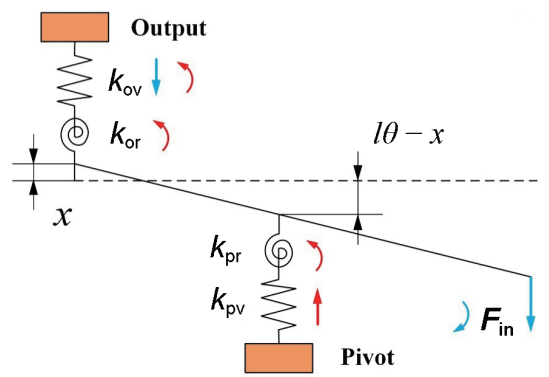

(b)

Fig. 2. (Color online) (a) Microlever before and after loading and (b) simplified model of the microlever used to derive the amplification factor.

$$
\begin{gathered}
\sum F=0, F_{\mathrm{in}}=k_{\mathrm{pv}}(l \theta-x)-k_{\mathrm{ov}} x, \\
\sum M=0, F_{\mathrm{in}} L=k_{\mathrm{pr}} \theta+k_{\mathrm{or}} \theta+k_{\mathrm{ov}} x l,
\end{gathered}
$$

where $F_{\text {in }}$ is the input force, $L$ is the distance between the input and the pivot, $l$ is the distance between the output and the pivot, $\theta$ is the rotation angle, $x$ is the displacement of the output, $k_{\mathrm{pv}}$ and $k_{\mathrm{ov}}$ are the vertical spring constants of the pivot and output, and $k_{\mathrm{pr}}$ and $k_{\text {or }}$ are the rotational spring constants of the pivot and output.

The direction of the output force $F_{\text {out }}$ is different from that of the vertical force of the output spring. According to the assumptions, the output force equals to the vertical spring constant multiplied by the displacement at the output

$$
F_{\text {out }}=-k_{\text {ov }} x
$$

The amplification of this microlever is the ratio of the output force to the input force:

$$
A=\frac{F_{\text {out }}}{F_{\text {in }}}=\frac{\frac{1}{k_{\mathrm{pv}}}\left(k_{\mathrm{pr}}+k_{\mathrm{or}}\right)-l L}{\left(\frac{1}{k_{\mathrm{pv}}}+\frac{1}{k_{\mathrm{ov}}}\right)\left(k_{\mathrm{pr}}+k_{\mathrm{or}}\right)+l^{2}},
$$

where $k_{\mathrm{pv}}=E w_{\mathrm{p}} t / l_{\mathrm{p}}, k_{\mathrm{ov}}=E w_{\mathrm{o}} t / l_{\mathrm{o}}, k_{\mathrm{pr}}=E t w_{\mathrm{p}}^{3} / 12 l_{\mathrm{p}}$, and $k_{\mathrm{or}}=E t w_{\mathrm{o}}^{3} / 12 l_{\mathrm{o}} ; E$ is Young's modulus, $t$ is the thickness of the device, and $l_{\mathrm{o}}$ and $l_{\mathrm{p}}$, and $w_{\mathrm{o}}$ and $w_{\mathrm{p}}$ are the lengths and widths of the output and pivot beams, respectively. From the expression of the amplification, we can observe when the vertical spring constant of the pivot, $k_{\mathrm{pv}}$, tends to infinity, when the rotational spring constants $k_{\mathrm{pr}}$ and $k_{\mathrm{or}}$ tend to zero, and when the amplification factor approaches $-L / l$, which is similar to the behavior of an ideal lever in the macroworld. 
The change in the spring constant of the beam springs under axial stress is investigated and the results of the static analysis are used to study the dynamic behavior of the resonator. Charge added to the capacitor plates induces an axial compression on the DETF resonator, which results in a corresponding downward shift in resonant frequency. The effect of this axially compressed frequency tuning is modelled in the following.

When adding the charge to the plates of the capacitor, the electrostatic force is

$$
F_{\mathrm{e}}=\frac{1}{2}\left|\frac{\partial C_{\mathrm{s}}}{\partial x}\right| V^{2}=\frac{1}{2}\left|\frac{\partial}{\partial x}\left(\frac{q^{2}}{C_{\mathrm{s}}}\right)\right|,
$$

where $C_{\mathrm{s}}$ is the total capacitance of the double charge sensing electrodes. The electrostatic force is amplified by the dual microlevers, and the axial force applied to the DETF can be expressed as

$$
F=|A| F_{\mathrm{e}}=\frac{|A| q^{2}}{2 \varepsilon_{0} w_{\mathrm{s}} t}
$$

where $w_{\mathrm{s}}$ is the width of the sensing electrodes. The resonant frequency of a generic device excited in its fundamental lateral mode is given by

$$
f_{0}=\frac{1}{2 \pi} \sqrt{\frac{k_{\mathrm{b}}}{M_{\mathrm{eff}}}},
$$

where $k_{\mathrm{b}}$ is the spring constant and $M_{\mathrm{eff}}$ is the effective mass of the device, including the beam and attached structures.

The tines of DETF are compressed when charge is added. The buckling of beams can be neglected owing to the tiny input charge to be detected. The spring constant is calculated using Euler-Bernoulli beam theory ${ }^{(17)}$ by simplifying the vibration of tines to a doubly clamped beam under axial force as shown in Fig. 3. The Euler beam equation is

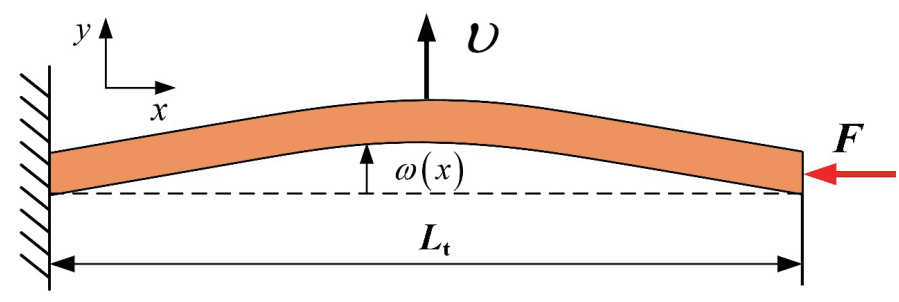

Fig. 3. (Color online) Schematic of the beam under axial stress; $v$ is the shear force. 


$$
E I \frac{\partial^{4} \omega}{\partial x^{4}}-F \frac{\partial^{2} \omega}{\partial x^{2}}=P
$$

where $I$ is the moment of inertia, $\omega$ is the deflection of the beam in the $y$-direction, and $P$ is the load distributed along the beam, which can be expressed using a Dirac delta function $[\delta(x)]: P(x)=v \delta\left(x-L_{\mathrm{t}} / 2\right)$, where $v$ is the shear force. The deflection is the largest in the middle of the beam. By solving the equation using boundary conditions, the spring constant $k_{\mathrm{b}}$ can be expressed as ${ }^{(18)}$

$$
k_{\mathrm{b}}(\gamma)=\frac{4 E I \gamma^{3}}{\gamma L_{\mathrm{t}}-4 \tanh \left(\gamma L_{\mathrm{t}} / 4\right)},
$$

where $L_{\mathrm{t}}$ is the length of each tine. Here, the parameter $\gamma$ is defined as $\gamma^{2}=F / E I$. The moment of inertia is given by $I=t w_{\mathrm{t}}^{3} / 12$, in which $w_{\mathrm{t}}$ is the width of each tine. Expanding eq. (9) as a Taylor series, $k_{\mathrm{b}}$ is rewritten as

$$
\begin{aligned}
k_{\mathrm{b}}(\gamma) & =\frac{192 E I}{L_{\mathrm{t}}^{3}}+\frac{24 E I}{5 L_{\mathrm{t}}} \gamma^{2}-\frac{E I L_{\mathrm{t}}}{700} \gamma^{4}+O\left(\gamma^{6}\right) \\
& \approx \frac{192 E I}{L_{\mathrm{t}}^{3}}\left(1+\frac{L_{\mathrm{t}}^{2}}{40} \gamma^{2}\right) .
\end{aligned}
$$

Substituting eq. (10) into eq. (7), we obtain the resonance frequency with axial force,

$$
f=f_{0} \sqrt{1+\frac{0.3 L_{\mathrm{t}}^{2}}{E t w_{\mathrm{t}}^{3}} F} .
$$

Expanding eq. (11) once again as a Taylor series, the frequency can be expressed as

$$
\begin{aligned}
f & =f_{0}\left[1+\frac{0.15 L_{\mathrm{t}}^{2} F}{E t w_{\mathrm{t}}^{3}}-\frac{0.01 L_{\mathrm{t}}^{4} F^{2}}{E^{2} t^{2} w_{\mathrm{t}}^{6}}+\frac{0.002 F^{3} L_{\mathrm{t}}^{6}}{E^{3} t^{3} w_{\mathrm{t}}^{9}}+O(F)\right] \\
& \approx f_{0}\left(1+\frac{0.15 L_{\mathrm{t}}^{2}}{E t w_{\mathrm{t}}^{3}} F\right) .
\end{aligned}
$$

The frequency shift as a function of electrostatic force is given by

$$
\Delta f=f-f_{0}=f_{0}\left(\frac{0.15 L_{\mathrm{t}}^{2}}{E t w_{\mathrm{t}}^{3}} F\right) .
$$

For the DETF, the axial force is equally distributed between the two tines and hence the frequency shift should be reduced by half. Substituting eq. (6) into eq. (13), we obtain the relationship between the resonance frequency shift and the input charge: 


$$
\Delta f=f_{0}\left(\frac{0.0375 L_{\mathrm{t}}^{2}|A|}{\varepsilon_{0} E t^{2} w_{\mathrm{t}}^{3} w_{\mathrm{s}}}\right) q^{2} .
$$

As we can see from eq. (14), the output frequency shift is proportional to the square of the input charge. The sensitivity is determined by the dimensions of the device and benefits from a thinner and narrower tine of the DETF. In addition, the amplification factor A plays an important role in enhancing the sensitivity. Owing to the manufacturing process, the minimum thickness and beam width are limited. Hence, improving the amplification factor using the optimized design is beneficial for obtaining considerable sensitivity.

\section{Simulation Results and Optimization}

In this work, finite element method (FEM) simulation by ANSYS was carried out to simulate the resonance frequency shift induced by the addition of charge to the plates of sensing capacitors. The interaction between the electrostatic force and resonant frequency of the mechanical structure was realized through pre-stress modal analysis. We apply the TRANS 126 element that converts the energy from an electrostatic domain into a structural domain. The element fully couples the electromechanical domains and represents a reduced-order model suitable for use in structural finite element analysis. The element has up to two degrees of freedom at each node: translation in the nodal $x, y$, or $z$ direction, and electric potential. In the simulation, we can calculate the corresponding voltages of different charge quantities using the expression $V=q / C_{\mathrm{s}}$. The element is suitable for simulating the electromechanical response of the MEMS resonator under electrostatic actuation. Static analysis with the pre-stress option was performed to calculate the amount of axial stress induced along the beams. A modal analysis was performed afterwards to calculate the shift in resonant frequency. A system-level simulation of the resonant charge sensor has been conducted in this study. The optimization study is carried out using this system-level simulation to achieve the maximum frequency shift.

The thickness of the whole structure has been fixed at $25 \mu \mathrm{m}$, which is determined by the thickness of the device layer in a silicon-on-insulator (SOI) wafer. The width and length of the DETF are critical dimensions (CDs). The wider and longer the tine, the higher the sensitivity. However, the long and narrow released beam tends to curl out-ofplane owing to intrinsic and surface stresses. For this reason, double $300 \times 4 \mu^{2}$ tines were used for the DETF in this design.

The important design step for the charge sensor has been the topological optimization of the microlevers. The lever arm must be sufficiently wide to maintain rigidity when bending. A long lever beam is beneficial in obtaining a larger lever ratio $(L / l)$. On the basis of the performance and fabrication limitations, a $300 \times 40 \mu \mathrm{m}^{2}$ lever arm was used.

To ensure the stability of the support and avoid the minimum line width limited by the fabrication process, the width of the output and pivot beams was chosen to be $4 \mu \mathrm{m}$. The critical dimensions needed to optimize by system-level simulation are the length of the output, $L_{\mathrm{o}}$, the length of the pivot, $L_{\mathrm{p}}$, and the distance between the center of the pivot and the output edge of the lever arm, $D_{\mathrm{p}}$. 


\subsection{Effects of the output and pivot beam lengths on the sensitivity}

The beam lengths of the microlever output and pivot are critical to the realization of the amplification function and the enhancement of sensitivity. It is necessary to make a careful study of the resonant frequency shift variation trend for different dimensions. When $D_{\mathrm{p}}$ is $30 \mu \mathrm{m}$, the frequency shift is shown in Fig. 4 as a function of output beam length under different pivot beam lengths with $200 \mathrm{fC}$ input charge.

From the trend, we can observe that the frequency shift increases with the increase in the length of the output beam. The variation remains stable when the length exceeds $60 \mu \mathrm{m}$. Obviously, the vertical and bending constants make it easier to transfer the force with a broad range of output beam lengths. However, a long beam induces instability when vibrating. The output beam length of $60 \mu \mathrm{m}$ is the optimized value. With increasing input charge, the variation is more pronounced. From Fig. 4, it can be seen that the variation in the length of the pivot has a weak effect on the frequency shift. In addition, an optimum pivot length between 20 and $30 \mu \mathrm{m}$ is obtained, but the frequency difference beweem these similar lengths is only $1 \mathrm{~Hz}$. Therefore, there is no need to determine the exact optimum pivot length.

\subsection{Effect of pivot position along lever beam length on sensitivity}

The simulation results shown in Fig. 5 provide good insight into the effect of the pivot position. For an ideal lever, the lever ratio should be as large as possible to obtain a high amplification factor. However, an optimized position exists for the pivot, because the amplification relies on the deflection of the compliant mechanisms. If the resisting arm is very short, the elastic energy loss becomes serious and the force does not transform to the output effectively. When the distance between the arm beam edge and the pivot center is $35 \mu \mathrm{m}$, the frequency shift is the largest.
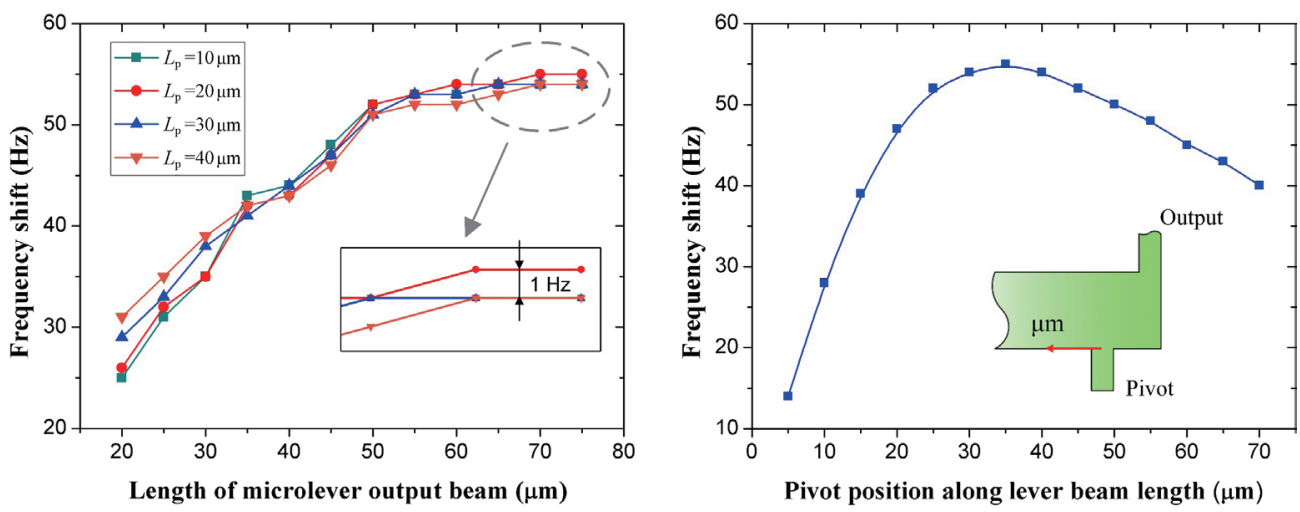

Fig. 4 (left). (Color online) System-level simulation results for a device with different lengths of microlever output and pivot beams when the input charge is $200 \mathrm{fC}$.

Fig. 5 (right). (Color online) System-level simulation results for a device with different pivot positions along lever beam length when the input charge is $200 \mathrm{fC}$. 
The optimized geometry based on the system-level simulation and other design parameters are presented in Table 1.

\subsection{Performance of the charge sensor}

The simulated natural frequency of the second mode is $169 \mathrm{kHz}$, which agrees well with the theoretical value (the two are typically within a fraction of $1.8 \%$ of each other). Figure 6(a) shows the out-of-phase mode of the sensor. The added microlevers remain static when the DETF element vibrates, playing an appropriate role of the anchor. Hence, the introduced compliant mechanisms do not affect the stability of the whole structure when the resonator vibrates.

The stress distribution with 10V DC bias on the driving electrodes and $200 \mathrm{fC}$ added to the charge input electrode is shown in Fig. 6(b). From this figure, we can observe that the output of the microlever exports an amplified stress and the deflections of the microlever are identical with the theoretical model. The harmonic response of the sensor is shown in Fig. 7. A corresponding downward shift in resonant frequency can be clearly observed after charge is added.

The relationship between the input charge and the resonant frequency shift is shown in Fig. 8. The simulation results match very well with the analytical solution for the proposed resonant charge sensor with microlevers. The simulated responsivity of

Table 1

Design parameters for the device.

\begin{tabular}{lc}
\hline Parameters & Value \\
\hline Device thickness $(\mu \mathrm{m})$ & 25 \\
DETF tine width $(\mu \mathrm{m})$ & 4 \\
DETF tine length $(\mu \mathrm{m})$ & 300 \\
Effective mass of tine $(\mathrm{ng})$ & 26.21 \\
Attached mass $(\mathrm{ng})$ & 121.39 \\
Effective stiffness of tine $(\mathrm{N} / \mathrm{m})$ & 161.2 \\
Resonant frequency $(\mathrm{kHz})$ & 166.326 \\
Lever beam width $(\mu \mathrm{m})$ & 40 \\
Lever beam length $(\mu \mathrm{m})$ & 300 \\
Pivot position along the lever $(\mu \mathrm{m})$ & 35 \\
Pivot width $(\mu \mathrm{m})$ & 4 \\
Pivot length $(\mu \mathrm{m})$ & 30 \\
Output width $(\mu \mathrm{m})$ & 4 \\
Output length $(\mu \mathrm{m})$ & 60 \\
Amplification of the microlever & -8.77 \\
Charge input capacitance $(\mathrm{fF})$ & 3.54 \\
\hline
\end{tabular}




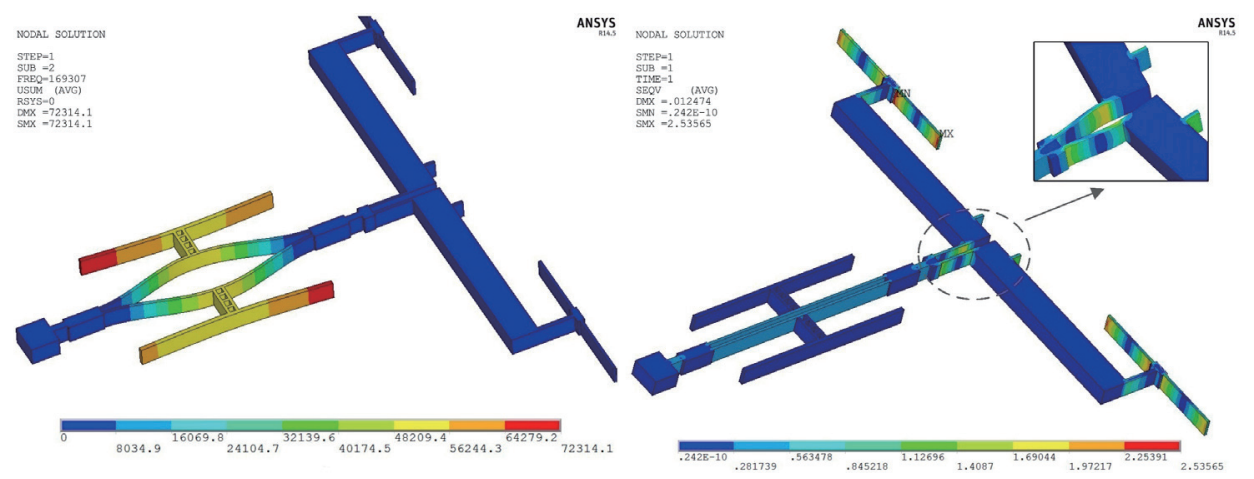

(a)

(b)

Fig. 6. (Color online) (a) Working mode shapes of the device and (b) stress distribution with 10 $\mathrm{V}$ DC on the driving electrode and $200 \mathrm{fC}$ on the charge input electrode.
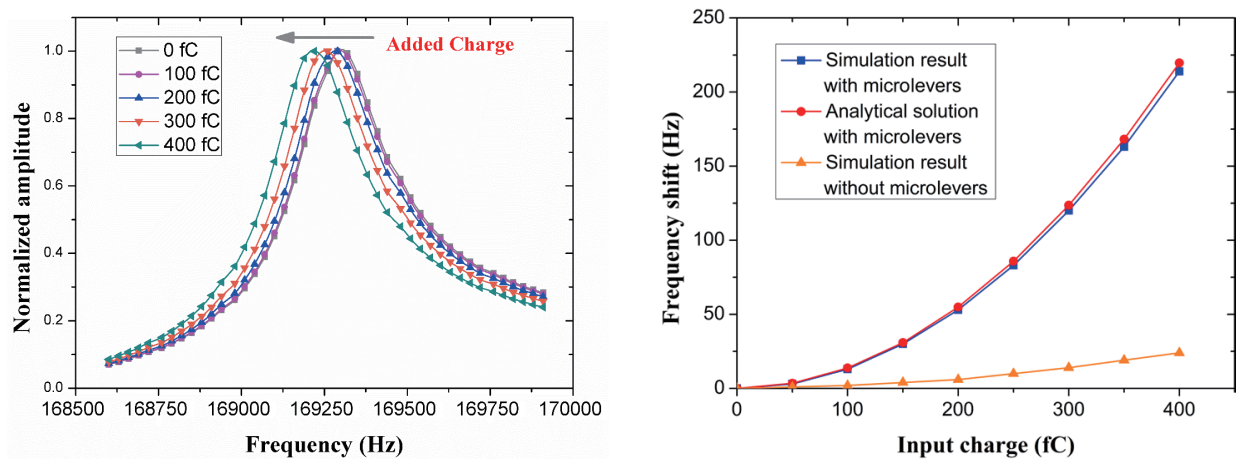

Fig. 7 (left). (Color online) Frequency response after harmonic simulation, showing the corresponding resonant frequency shift with charge loading.

Fig. 8 (right). (Color online) Simulation and analytical curves relating input charge and frequency shift.

the charge sensor is $1.38 \times 10^{-3} \mathrm{~Hz} / \mathrm{fC}^{2}$. The responsivity of the analytical solution is slightly larger than the simulation results because some deflections are neglected in the assumptions in the simplified model. From the figure, we also find that the frequency shift of the sensor with microlevers is much larger than that without microlevers. Obviously, the responsivity is enhanced through the introduction of compliant mechanisms. In addition, the output of the charge sensor is a change in frequency, which is easy to measure and robust against noise. 


\section{Fabrication}

The device was fabricated by a commercially available SOIMUMPs process. ${ }^{(19)}$ Figure 9 presents the illustrated summary of this process. The process starts with a SOI wafer, which is constructed with a highly doped $25-\mu \mathrm{m}$-thick n-type device layer, a 2 $\mu \mathrm{m}$ oxide layer and a $400 \mu \mathrm{m}$ substrate layer. The top silicon layer is lithographically patterned and is subjected to deep reactive ion etching (DRIE) to obtain a fixed and movable mechanical structure from the front side following metallization for defining the bond pads as shown in Figs. 9(a) and 9(b). Figure 9(c) shows that a polyimide protective coat is subsequently applied to the front side. A trench underneath the suspended structure is etched through the substrate from the back by DRIE [Fig. 9(d)], exposing the underlying buried oxide layer that is then removed by wet HF etching as shown in Fig. 9(e). The suspended structure is then fully released when the front-side polyimide protective coat is removed as shown in Fig. 9(f). These trenches not only release the movable parts but also reduce the substrate parasitic capacitance. The fabricated device is shown in Fig. 10.

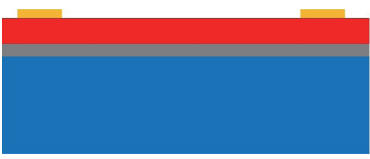

(a)

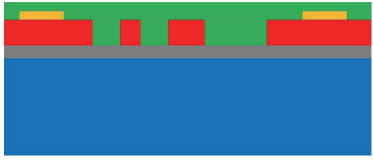

(c)

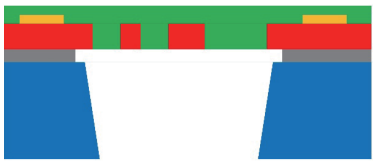

(e)

SOI device layer

SOI handle layer

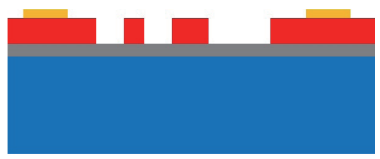

(b)

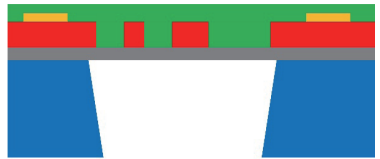

(d)

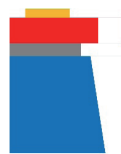

(f)

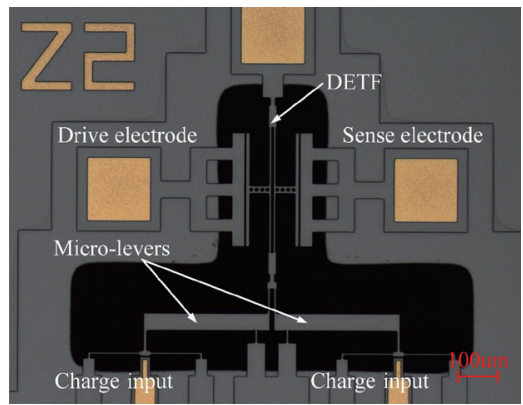

Fig. 9 (left). (Color online) Fabrication process by the SOIMUMPs process.

Fig. 10 (right). (Color online) Optical micrograph of the fabricated device with dual microlevers. 


\section{Conclusions}

The design, modeling, system-level simulation and fabrication of a novel micromachined resonant charge sensor with dual microlevers are presented in this paper. System-level FEM simulation has been carried out to predict the performance of the sensor and optimize some important parameters, which show good agreement with theoretical solutions. System-level simulation is usually desired for accurate optimization and predicting performance before fabrication. The modeling method introduced here can be extended to the modeling of other resonating sensors. The simulated responsivity is $1.38 \times 10^{-3} \mathrm{~Hz} / \mathrm{fC}^{2}$ was enhanced 8 -fold after the introduction of microlevers. We have found that the microlevers do not affect the stability and electronic noise level of the sensor. The device reported in this paper has important advantages such as small size, easy implementation, and high resolution. In addition, the sensor can be applied as a handy and low-cost electrometer and integrated to other systems for detection.

\section{Acknowledgements}

This work is supported by the "Zhejiang Provincial Natural Science Foundation of China (No. LY14E050018)", the "National Natural Science Foundation of China (No. 51475423 and No. 51275465)", and the "Zhejiang Open Foundation of the Most Important Subjects".

\section{References}

1 F. J-Fatokun, L. Morawska, M. Jamriska and E. Jayaratne: Atmos. Environ. 42 (2008) 8827.

2 T. Ireland, N. Schram, P. Holden, P. Lanc, J. Ávila and R. Armstrong: Int. J. Mass Spectrom. 359 (2014) 26.

3 G. Jaramillo, C. Buffa, M. Li, F. J. Brechtel, G. Langfelder and D. A. Horsley: IEEE Sens. J. 13 (2013) 2993.

4 C. Calle, J. Mantovani, C. Buhler, E. Groop, M. Buehler and A. Nowicki: J. Electrostat. 61 (2004) 245.

5 G. Zimmerli, T. Eiles, R. Kautz and J. M. Martinis: Appl. Phys. Lett. 61 (1992) 237.

6 P. S. Riehl, K. L. Scott, R. S. Muller, R. T. Howe and J. A. Yasaitis: J. Microelectromech. Syst. 12 (2003) 577.

7 J. Lee, Y. Zhu and A. Seshia: J. Micromech. Microeng. 18 (2008) 025033.

8 A. N. Cleland and M. L. Roukes: Nature 392 (1998) 160.

9 H. Krömmer, A. Erbe, A. Tilke, S. Manus and R. Blick: EPL (Europhysics Letters) 50 (2000) 101.

10 J.-Y. Lee, B. Bahreyni, Y. Zhu and A. A. Seshia: IEEE Electron Device Letters 29 (2008) 701.

11 S. Khan and G. Ananthasuresh: J. Microelectromech. Syst. 23 (2014) 871.

12 I. Zeimpekis, I. Sari and M. Kraft: J. Microelectromech. Syst. 21 (2012) 1032.

13 C. B. Pedersen and A. A. Seshia: J. Micromech. Microeng. 14 (2004) 1281.

14 S. X. Su: Compliant leverage mechanism design for MEMS applications (Ph.D. Dissertation, University of California, Berkeley, 2001).

15 X. Liu, J. Tong and Y. Sun: Smart Mater. Struct. 16 (2007) 1742.

16 J.-C. Tsai and M. C. Wu: J. Microelectromech. Syst. 15 (2006) 1209.

17 S. D. Senturia: Microsystem Design (Kluwer Academic Publishers, Boston, 2001) Vol. 3, 201.

18 B. Bahreyni and C. Shafai: IEEE Sens. J. 7 (2007) 1326.

19 A. Cowen, G. Hames, D. Monk, S. Wilcenski and B. Hardy: SOIMUMPS Design Handbook, rev. 6.0, MEMSCAP Inc. (2011). 